\title{
Effect of Hijikia fusiforme extracts on degenerative osteoarthritis in vitro and in vivo models
}

\author{
Han Ol Kwon', Minhee Lee', Ok-Kyung Kim', Yejin Ha ${ }^{1}$, Woojin Jun ${ }^{2}$ and Jeongmin Lee ${ }^{1 \S}$ \\ ${ }^{1}$ Department of Medical Nutrition, Kyung Hee University, 1732, Deogyeong-daero, Giheung-gu, Yongin 17104, Korea \\ ${ }^{2}$ Division of Food and Nutritional Science, Chonnam National University, Gwangju 61186, Korea
}

BACKGROUND/OBJECTIVES: The inhibitory effect of Hijikia fusiforme (HF) extracts on degenerative osteoarthritis was examined in primary cultured rat cartilage cells and a monosodium iodoacetate (MIA)-induced osteoarthritis rat model.

MATERIALS/METHODS: In vitro, cell survival and the expression of matrix metalloproteinases (MMPs), collagen type I, collagen type II, aggrecan, and tissue inhibitor of metalloproteinases (TIMPs) was measured after $\mathrm{H}_{2} \mathrm{O}_{2}(800 \mu \mathrm{M}, 2 \mathrm{hr})$ treatment in primary chondrocytes. In vivo animal study, osteoarthritis was induced by intra-articular injection of MIA into knee joints of rats, and then RH500, HFE250 and HFE500 were administered orally once a day for 28 days. To determine the anti-inflammatory effects of HFE, nitric oxide (NO), prostaglandin $E_{2}\left(P_{G}\right)$ expression were measured. In addition, real-time PCR was performed to measure the genetic expression of MMPs, collagen type I, collagen type II, aggrecan, and TIMPs.

RESULTS: In the in vitro assay, cell survival after $\mathrm{H}_{2} \mathrm{O}_{2}$ treatment was increased by HFE extract (20\% EtOH). In addition, anabolic factors (genetic expression of collagen type I, II, and aggrecan) were increased by HFE extract (20\% EtOH). However, the genetic expression of MMP-3 and 7, known as catabolic factors were significantly inhibited by treatment with HFE extract (20\% EtOH). In the in vivo assay, anabolic factors (genetic expression of collagen type I, II, aggrecan, and TIMPs) were increased by oral administration of HFE extract. However, the genetic expression of MMP-3 and 7, known as catabolic factors, and production of $\mathrm{NO}$ and $\mathrm{PGE}_{2}$ were significantly inhibited by treatment with oral administration of HFE extract.

CONCLUSION: HFE extract inhibited articular cartilage degeneration through preventing extracellular matrix degradation and chondrocyte injury.

Nutrition Research and Practice 2016;10(3):265-273; doi:10.4162/nrp.2016.10.3.265; pISSN 1976-1457 elSSN 2005-6168

Keywords: Chondrocyte, Collagen, MMP, TIMP

\section{INTRODUCTION}

According to the statistics of the National Health and Nutrition Examination Survey, the prevalence of arthritis in the Republic of Korea was $37.3 \%$ in the over 50 years of age, and differed depending on gender and age [1,2]. Osteoarthritis, the most common arthritis, is a degenerative joint disease characterized by joint pain and swelling through gradual loss of articular cartilage. Age, sex, family history, joint stress, and obesity are major contributing factors to the development of osteoarthritis. It can lower the quality of life by inhibiting daily activities and causing movement disorders [3].

Degenerative osteoarthritis occurs in the damaged joint when chondrocytes, joint cartilage cells, activate the synthesis of the matrix proteinase components and degradation of cartilage matrix. Factors associated with activation of chondrocytes are protein hydrolysis enzyme (proteolytic enzymes), cytokines, and nitric oxide (NO). Pro-inflammatory cytokines cause the expression of proteases such as collagenase, aggrecanase, and matrix metalloproteinases (MMPs), tissue inhibitor of metalloproteinases (TIMPs), and then the activation of protease induces the degradation of the collagen matrix of cartilage and impairment of procollagen synthesis. In addition, pro-inflammatory cytokines plays an extremely important role in the development of inflammation by production of prostaglandin $\mathrm{E}_{2}\left(\mathrm{PGE}_{2}\right)$ and $\mathrm{NO}$ [4-7].

Therefore, pharmacologic interventions have focused on anti-inflammation and pain relief by non-steroidal anti-inflammatory drug (NSAID) and corticosteroids. However, due to side effects of the pharmacologic interventions, the demand for health functional food is increasing rapidly. Accordingly, the market for health functional food associated with osteoarthritis has been persistently maintained, expectations of finding a new functionality of health functional food ingredients have

\footnotetext{
This research was financially supported by the Korea Institute for Advancement of Technology (KIAT, 20141482) through the research and development for regional industry.

This work was supported by a grant from Kyung Hee University (KHU-20120817).

${ }^{\S}$ Corresponding Author: Jeongmin Lee, Tel. 82-31-201-3838, Fax. 82-31-201-2114, Email. Jlee2007@khu.ac.kr

Received: August 26, 2015, Revised: November 26, 2015, Accepted: January 6, 2016

This is an Open Access article distributed under the terms of the Creative Commons Attribution Non-Commercial License (http://creativecommons.org/licenses/by-nc/3.0/) which permits unrestricted non-commercial use, distribution, and reproduction in any medium, provided the original work is properly cited.
} 
increased [8-11].

Hijikia fusiforme is brown seaweed widely distributed in Japan, Korea, and China. [12]. Laminaran and fucoidan are the major constituents of Hijikia fusiforme, which has biological effects of blood coagulation, immune regulation, prevention of cholesterol deposits in blood vessels, and anti-cancer [12-16]. Jeong et al. [16] reported that Hijikia fusiforme induced NO production and enhancement of pro-inflammatory cytokines in Raw 264.7 cells and splenocytes. However, improving in osteoarthritis requires the opposite for anti-inflammation, such as reductions of NO and pro-inflammatory cytokines. In this study, the preventive effects of Hijikia fusiforme against osteoarthritis induced by monosodium iodoacetate (MIA) injection in rats were examined. The effects of oral administration of Hijikia fusiforme on anti-inflammation were examined by measuring levels of pro-inflammatory cytokine, nitric oxide, $\mathrm{PGE}_{2}$, and gene expression.

\section{MATERIALS AND METHODS}

\section{Preparation of Hijikia fusiforme}

Fresh Hijikia fusiforme was purchased from a commercial supplier in Wando, Republic of Korea. The fresh Hijikia fusiforme (60 kg) was dried for 3 days at room temperature after washing in tap water, yielding $6 \mathrm{~kg}$ of dried Hijikia fusiforme. The dried Hijikia fusiforme (HF) was extracted with 20 volumes of water at $100^{\circ} \mathrm{C}$ for $4 \mathrm{~h}$, and $30 \%$ or $80 \%$ ethanol at $20^{\circ} \mathrm{C}$ for $12 \mathrm{~h}$ in a reflux apparatus. Extracts were filtered using Whatman (Maidstone, United Kingdom) paper (NO. 2) and concentrated at $50^{\circ} \mathrm{C}$ using rotary evaporators under reduced pressure. The concentrate was freeze-dried, and yields of Hijikia fusiforme water extract (HW), Hijikia fusiforme $20 \%$ ethanol extract (ET20), and Hijikia fusiforme $50 \%$ ethanol extract (ET50) were $6.5 \%$, $4.8 \%$, and $3.6 \%$, respectively. Each extract was stored in a tight and light-protected container at $-20^{\circ} \mathrm{C}$ until used.

\section{Primary culture of chondrocyte}

The study was approved by the Institutional Animal Care and Use Committee of Kyung Hee University (KHUASP(SE)-15-020). Six week male Sprague-Dawley rats (160-180 g) were sacrificed by cervical dislocation under ether anesthesia and both tibiae were dissected free. Cartilage were separately sliced and incubated at room temperature in Hank's balanced salt solution (HBSS, Hyclone Laboratories, Logan, Utah, USA) containing 2 $\mathrm{mg} / \mathrm{mL}$ collagenase (SERVA, Heidelberg, Germany), and overnight in a shaker at $100 \mathrm{rpm}$. The chondrocytes obtained by this method were seeded on a 75T flask in DMEM medium (Hyclone Laboratories, Logan, Utah, USA) with $10 \%$ fetal bovine serum (FBS, Hyclone Laboratories, Logan, Utah, USA), $50 \mu \mathrm{L}$ gentamycin sulfate (Hyclone Laboratories, Logan, Utah, USA) and cells were cultured in a $5 \% \mathrm{CO}_{2}$ incubator at $35^{\circ} \mathrm{C}$.

\section{Cell viability}

Cell viability was assessed using 3-(4,5-dimethylthiazol-2yl-)2,5-diphenyl tetrazolium bromide (MTT) (Sigma Aldrich, Sigma, St. Louis, MO, USA). Primary chondrocytes were cultured with extracts at various concentrations. After incubation for $24 \mathrm{~h}$, $20 \mu \mathrm{L}$ of MTT solution ( $5 \mathrm{mg} / \mathrm{mL}$ in PBS as stock solution) were added to each well, and the cells were incubated again at $37^{\circ} \mathrm{C}$ for $3 \mathrm{~h}$. The supernatants were removed, and DMSO (200 $\mu \mathrm{L})$ was then added to each well. The plates were read at $560 \mathrm{~nm}$ to obtain the percentage of viable cells.

\section{Measurement of gene expression in primary chondrocytes}

Chondrocytes were plated at a density of $5 \times 10^{5}$ cells/well in 6-well plate and incubated for $24 \mathrm{~h}$. The HF extracts $(0-1,000$ $\mu \mathrm{g} / \mathrm{mL}$ ) were applied and the cells stimulated by the addition of LPS $50 \mu \mathrm{g} / \mathrm{mL}$ or $\mathrm{H}_{2} \mathrm{O}_{2} 800 \mu \mathrm{M}$. After incubation for $24 \mathrm{~h}$, mRNA was extracted from seeding cells using the RNeasy Mini Kit (QIAGEN, Maryland, USA) and CDNA was prepared using the iScript $^{\text {TM }}$ cDNA Synthesis kit (BIORAD, Hercules, CA, USA). Real-Time PCR (Applied Biosystems, Foster City, CA, USA) was performed using the CDNA (1 $\mu \mathrm{L})$ with the SYBR Green PCR Master Mix (iQ SYBR Green Supermix, BIORAD, Hercules, CA, USA). The CDNA was amplified for 40 cycles of denaturation $\left(95^{\circ} \mathrm{C}\right.$ for $\left.30 \mathrm{~s}\right)$, annealing $\left(58^{\circ} \mathrm{C}\right.$ for $\left.30 \mathrm{~s}\right)$, and extension $\left(72^{\circ} \mathrm{C}\right.$ for 45 s) using the following primers: GAPDH forward primer 5'-TGG CCT CCA AGG AGT AAG AAA C-3', reverse primer 5'-CAG CAA CTG AGG GCC TCT CT-3'; Aggrecan forward primer 5'-GAA GTG GCG TCC AAA CCA A-3', reverse primer 5'-CGT TCC ATT CAC CCC TCT CA-3'; Collagen Type I forward primer 5'-GAG CGG AGA GTA CTG GAT CGA-3', reverse primer 5'-CTG ACC TGT CTC CAT GTT GCA-3'; Collagen Type II forward primer 5'-GCA ACA GCA GGT TCA CGT ACA-3', reverse primer 5'-TCG GTA CTC GAT GAT GGT CTT G-3'; TIMP-1 forward primer 5'-AAG GGC TAC CAG AGC GAT CA, reverse primer 5'-ATC GAG ACC CCA AGG TAT TGC-3'; TIMP-3 forward primer 5'-GAC CGA CAT GCT CTC CAA TIT C-3', reverse primer 5'-GCT GCA GTA GCC ACC CTT CT-3'; MMP-3 forward primer 5'-GAG TGT GGA TTC TGC CAT TGA G-3', reverse primer 5'-TTA TGT CAG CCT CTC CTT CAG AGA-3'; MMP-7 forward primer 5'-ACT CTA GGC CAT GCC TाT GC-3', reverse primer 5-'CCA TCC GTC CAG TAC TCA TCC T-3'; TNF-a forward primer 5'-ACA AGG CTG CCC CGA CTA T-3', reverse primer 5'-CTC CTG GTA TGA AGT GGC AAA TC-3'; IL-6 forward primer 5'-GCC CTT CAG GAA CAG CTA TGA-3'; reverse primer 5'-TGT CAA CAA CAT CAG TCC CAA GA-3'. Data analysis of real-time RT-PCR results and calculations of the relative quantitation were performed using the 7500 System SDS software version 1.3.1 (Applied Biosystems, Foster City, CA, USA).

\section{Animals and treatment}

Male Sprague-Dawley rats (6 weeks old) were obtained from Japan SLC, Inc. (Hamamatsu, Japan) and housed at the animal facilities of Kyung Hee University (Yongin, Republic of Korea). The study was approved by the Institutional Animal Care and Use Committee of Kyung Hee University (KHUASP(SE)-15-020). After three days of accommodation, the rats were randomly assigned to five groups (8 rats per group); 1 ) Normal group

Table 1. Experimental design animals ( $n=8 /$ group)

\begin{tabular}{lccc}
\hline Groups & Induced arthritis & Dietary administration & Orally administration \\
\hline Normal & - & AIN 93G diet & \\
Sham & + & AIN 93G diet & \\
RH500 & + & AIN 93G diet & Rose Hip $500 \mathrm{mg} / \mathrm{kg}$ \\
HFE250 & + & AIN 93G diet & Hijikia $250 \mathrm{mg} / \mathrm{kg}$ \\
HFE500 & + & AIN 93G diet & Hijikia $500 \mathrm{mg} / \mathrm{kg}$ \\
\hline
\end{tabular}


(wild type) with AIN-93G rodent diet, 2) arthritis group (sham) with AIN-93G rodent diet, 3) arthritis group (RH) with AIN-93G + Rose Hip extract 500 mg/kg/bw, 4) arthritis group (HFE250) with AIN-93G + Hijikia fusiforme $20 \%$ ethanol extract 250 $\mathrm{mg} / \mathrm{kg} / \mathrm{bw}, 5$ ) arthritis group (HFE500) with AIN-93G + Hijikia fusiforme $20 \%$ ethanol extract $500 \mathrm{mg} / \mathrm{kg} / \mathrm{bw}$. Three days after income, their respective sample was administered orally for 26 days (Table 1).

\section{Induction of osteoarthritis}

Male Sprague-Dawley rats (SLC Inc., Hamamatsu, Japan) weighing $200-250 \mathrm{~g}$ at the time of induction were used. Animals were anaesthetized using isofluorane and a single injection of $50 \mu \mathrm{L}$ sterile $0.9 \%$ saline containing $3 \mathrm{mg}$ of monosodiumiodoacetate (MIA, Sigma) was injected through the articular cavity using a $27 \mathrm{G}$ needle. Normal group injections used $0.9 \%$ saline only. Rats were sacrificed by cervical dislocation on day 24 post-MIA injection.

\section{Nitric oxide (NO) production}

Serum samples were obtained from rats by aspirating retroorbital blood on day 24 post-MIA injection. All samples were stored at $-80^{\circ} \mathrm{C}$ until used. A spectrophotometric method based on the Griess reaction with sodium nitrite as standard was used. Briefly, $50 \mu \mathrm{L}$ of serums were mixed with $50 \mu \mathrm{L}$ of Griess reagent (Sigma-aldrich, St Louis, Mo, USA), followed by incubation for $5 \mathrm{~min}$ at $37^{\circ} \mathrm{C}$. Optical density was measured at $540 \mathrm{~nm}$ using an ELISA reader (VERSAMAXSL-20 Molecular Devices, Korea).

\section{Prostaglandin $E_{2}\left(P G E_{2}\right)$ production}

Serum samples were obtained from rats by aspirating retro-orbital blood on day 24 post-MIA injection. All samples were stored at $-80^{\circ} \mathrm{C}$ until used. Serum level of $\mathrm{PGE}_{2}$ was determined using ELISA kits (KGE004B, R\&D system, Minneapolis, $M N, U S A)$, according to the manufacturer's instructions.

Measurement of gene expression in osteoarthritis rat cartilage mRNA was extracted from MIA-induced arthritis rat articular cartilage using the RNeasy Mini Kit (QIAGEN, Maryland, USA) and $C D N A$ was prepared using the iScript ${ }^{\mathrm{TM}}$ CDNA Synthesis kit (BIORAD, Hercules, CA, USA). Real-Time PCR (Applied Biosystems, Foster City, CA, USA) was performed using the CDNA $(1 \mu \mathrm{L})$ with the SYBR Green PCR Master Mix (iQ SYBR Green Supermix, BIORAD, Hercules, CA, USA). The CDNA was amplified for 40 cycles of denaturation $\left(95^{\circ} \mathrm{C}\right.$ for $\left.30 \mathrm{~s}\right)$, annealing $\left(58^{\circ} \mathrm{C}\right.$ for 30 s), and extension $\left(72^{\circ} \mathrm{C}\right.$ for $\left.45 \mathrm{~s}\right)$ using the following primers: GAPDH forward primer 5'-TGG CCT CCA AGG AGT AAG AAA C-3', reverse primer 5'-CAG CAA CTG AGG GCC TCT CT-3'; Aggrecan forward primer 5'-GAA GTG GCG TCC AAA CCA A-3', reverse primer 5'-CGT TCC ATT CAC CCC TCT CA-3'; Collagen Type I forward primer 5'-GAG CGG AGA GTA CTG GAT CGA-3', reverse primer 5'-CTG ACC TGT CTC CAT GTT GCA-3'; Collagen Type II forward primer 5'-GCA ACA GCA GGT TCA CGT ACA-3', reverse primer 5'-TCG GTA CTC GAT GAT GGT CTT G-3'; MMP-3 forward primer 5'-GAG TGT GGA TTC TGC CAT TGA G-3', reverse primer 5'-TTA TGT CAG CCT CTC CTT CAG AGA-3'; MMP-7 forward primer 5'-ACT CTA GGC CAT GCC TIT GC-3', reverse primer 5-'CCA TCC GTC CAG TAC TCA TCC T-3'. Data analysis of real-time RT-PCR results and calculations of the relative quantitation were performed using the 7500 System SDS software version 1.3.1 (Applied Biosystems, Foster City, CA, USA).

\section{Statistical analysis}

The experimental results are presented as the means $\pm S D$. The significance of treatment effects was assessed using Duncan's multiple range tests following one-way analysis of variance (ANOVA) using the SAS 9.2 version software (SAS Institute Inc., Cary, NC, USA). $P<0.05$ was considered to indicate a statistically significant difference.

\section{RESULTS}

Effects of the Hijikia fusiforme extracts on the cell viability of chondrocytes

The HW and ET20 did not affect the chondrocyte viability, but the viability of ET50- treated chondrocytes was significantly decreased at $600 \mu \mathrm{g} / \mathrm{mL}(P<0.05)$ (Fig. 1). Therefore, we decided to examine the effects of HW $0-1,000 \mu \mathrm{g} / \mathrm{mL}$, ET 0-1,000

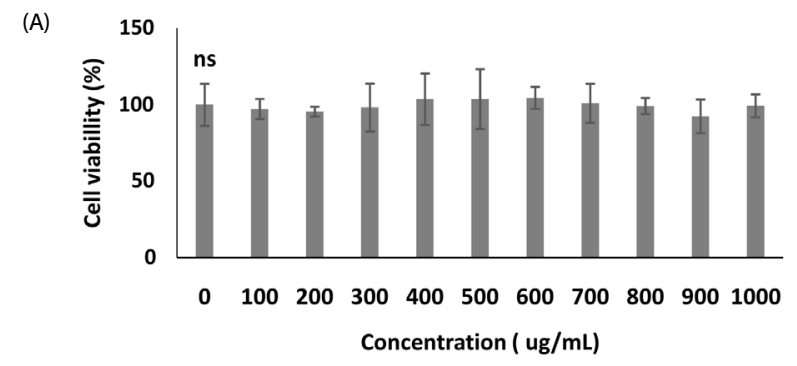

(B)
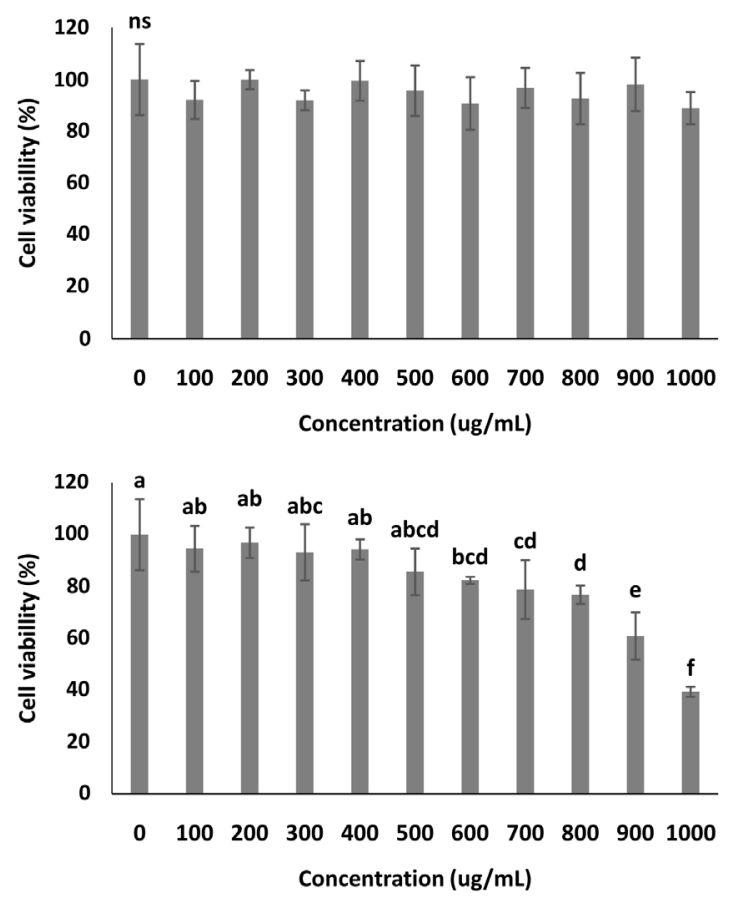

Fig. 1. Cell viability of primary cultured rat cartilage cells, determined by MTT reagent, with treatment of $\mathrm{HF}$ extracts at various concentrations. (A) Hot water extract $100-1,000 \mu \mathrm{g} / \mathrm{mL}$, (B) $20 \%$ EtOH extract $100-1,000 \mu \mathrm{g} / \mathrm{mL}$, (C) $50 \%$ EtOH extract $100-1,000 \mu \mathrm{g} / \mathrm{mL}$. Data are expressed as mean $\pm \mathrm{SD}(\mathrm{n}=3)$. Significant differences were determined using Duncan's multiple range test at $P<0.05$. 


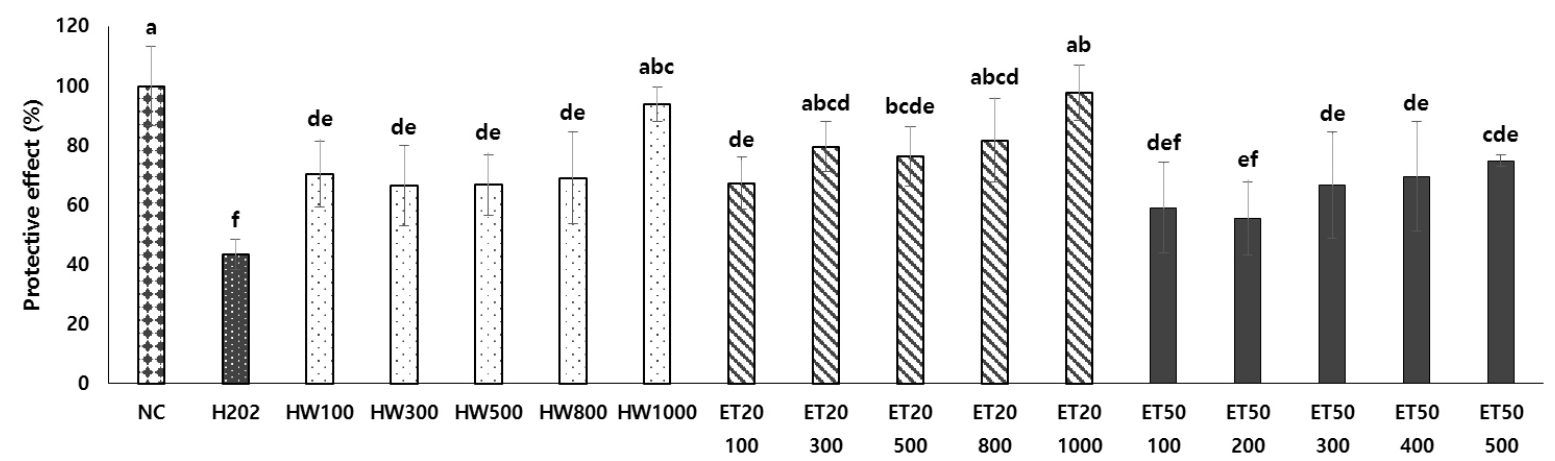

Fig. 2. Effect of $\mathrm{HF}$ extracts on survival against $800 \mu \mathrm{M} \mathrm{H} \mathrm{O}_{2}$ treatment. (A) Hot water extract 100-1,000 $\mu \mathrm{g} / \mathrm{mL}$, (B) $20 \%$ EtOH extract $100-1,000 \mu \mathrm{g} / \mathrm{mL}$, (C) $50 \%$ EtOH extract $100-1,000 \mu \mathrm{g} / \mathrm{mL}$. Data are expressed as mean $\pm \mathrm{SD}(\mathrm{n}=3)$. Significant differences were determined using Duncan's multiple range test at $P<0.05$.

(A)

LPS $50 \mu \mathrm{g} / \mathrm{mL}$

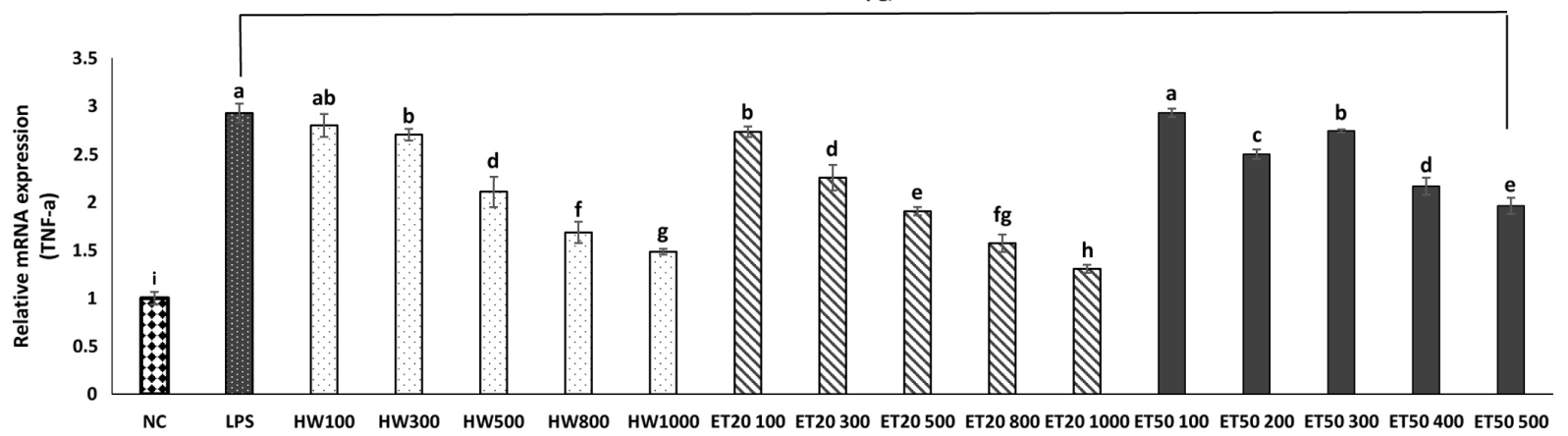

(B)

LPS $50 \mu \mathrm{g} / \mathrm{mL}$

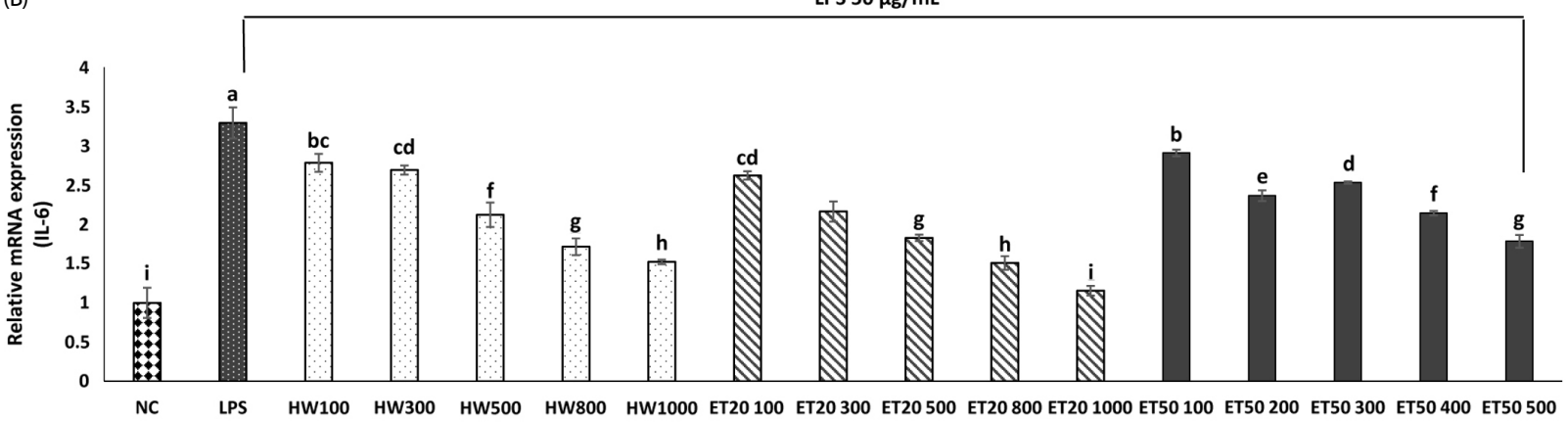

Fig. 3. Effect of HF extracts on genetic expression of cytokines in primary cultured cells. (A) TNF-a, (B) IL-6. HW; Hot water extract 100-1,000 $\mu \mathrm{g} / \mathrm{mL}$, ET20; 20\% EtOH extract 100-1,000 $\mu \mathrm{g} / \mathrm{mL}$, ET50; $50 \% \mathrm{EtOH}$ extract 100-1,000 $\mu \mathrm{g} / \mathrm{mL}$. Data are expressed as mean \pm SD $(n=3)$. Significant differences were determined using Duncan's multiple range test at $P<0.05$.

$\mu \mathrm{g} / \mathrm{mL}$, and ET50 0-500 $\mu \mathrm{g} / \mathrm{mL}$.

Protective effects of the Hijikia fusiforme extracts on $\mathrm{H}_{2} \mathrm{O}_{2}$-induced chondrocytes

The $\mathrm{H}_{2} \mathrm{O}_{2}$-induced chondrocytes showed significantly reduced cell viability to approximately $43.3 \%$ of normal chondrocyte viability. In the HW, ET20, and ET50, cell viability was significantly increased compared with that of the $\mathrm{H}_{2} \mathrm{O}_{2}$-induced chondrocyte control group $(P<0.05)$ (Fig. 2).

Effects of the Hijikia fusiforme extracts on the expression of TNF- $a$ and IL-6 in chondrocytes

We found that Hijikia fusiforme inhibited LPS-mediated TNF-a and IL- 6 gene expression in chondrocytes. HW, ET20, and ET50 inhibited TNF- $a$ and IL-6 expression in a dose-dependent manner. In particular, TNF-a expression in 1,000 $\mu \mathrm{g} / \mathrm{mL}$ ET20-treated cells was significantly decreased by $1.30 \pm 0.04$ compared with LPS treated cells $(2.93 \pm 0.10)(P<0.05)$ (Fig. $3 \mathrm{~A})$. In addition, IL-6 expression in $1,000 \mu \mathrm{g} / \mathrm{mL}$ ET20-treated cells also was significantly decreased by $1.15 \pm 0.06$ compared with LPS treated cells $(3.29 \pm 0.20)(P<0.05)$ (Fig. 3B).

Effects of the Hijikia fusiforme extracts on genetic expression of anabolic and catabolic factors in chondrocytes

We examined whether a Hijikia fusiforme inhibited $\mathrm{H}_{2} \mathrm{O}_{2}$ mediated type I collagen, type II collagen, aggrecan, TIMP-1, TIMP-3, MMP-3, and MMP-7 expression in chondrocytes. Expression of type I collagen, type II collagen, aggrecan, TIMP-1, 
(A)

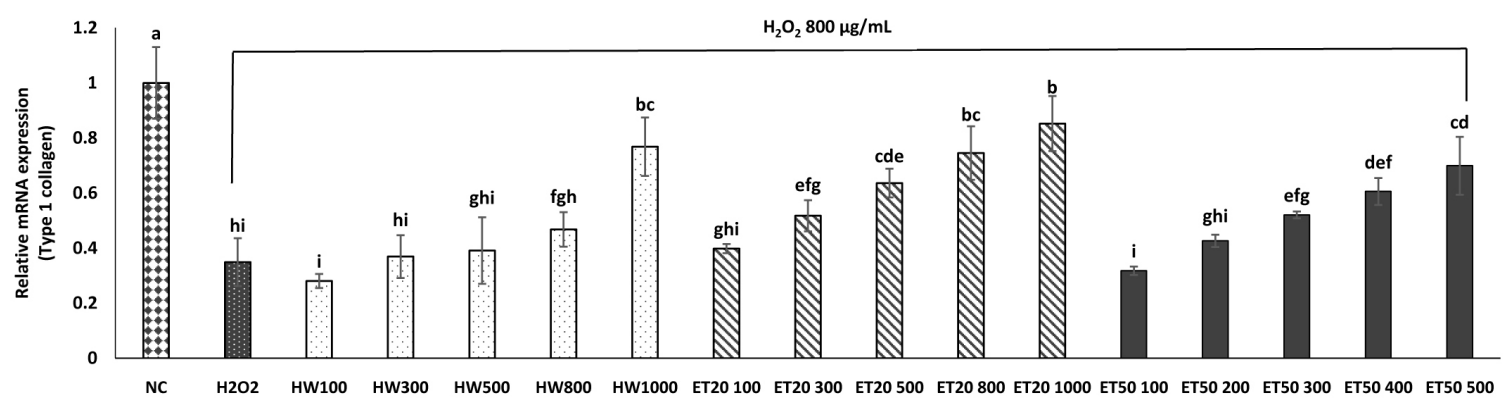

(B)

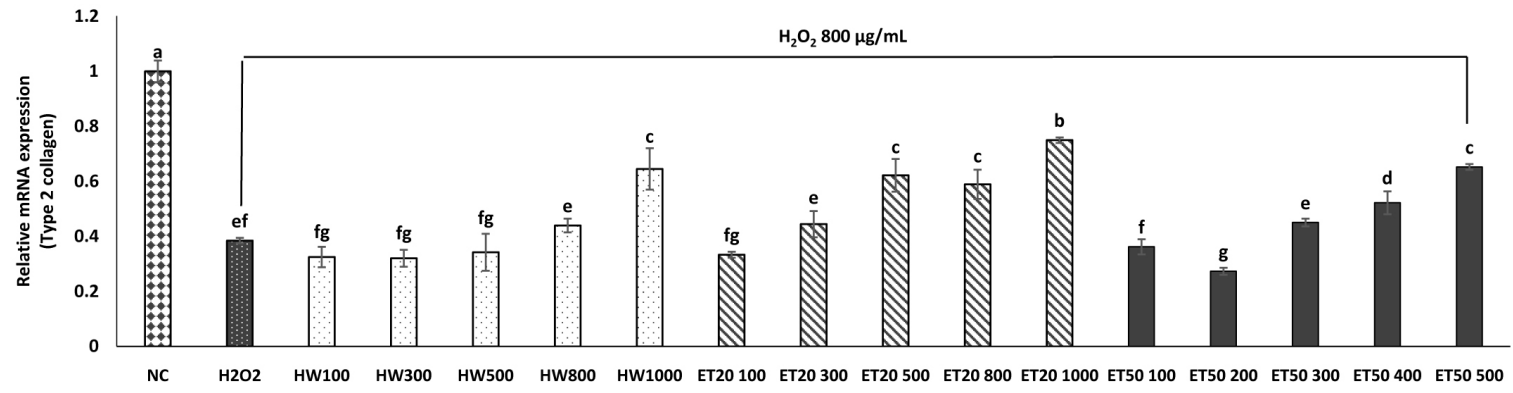

(C)

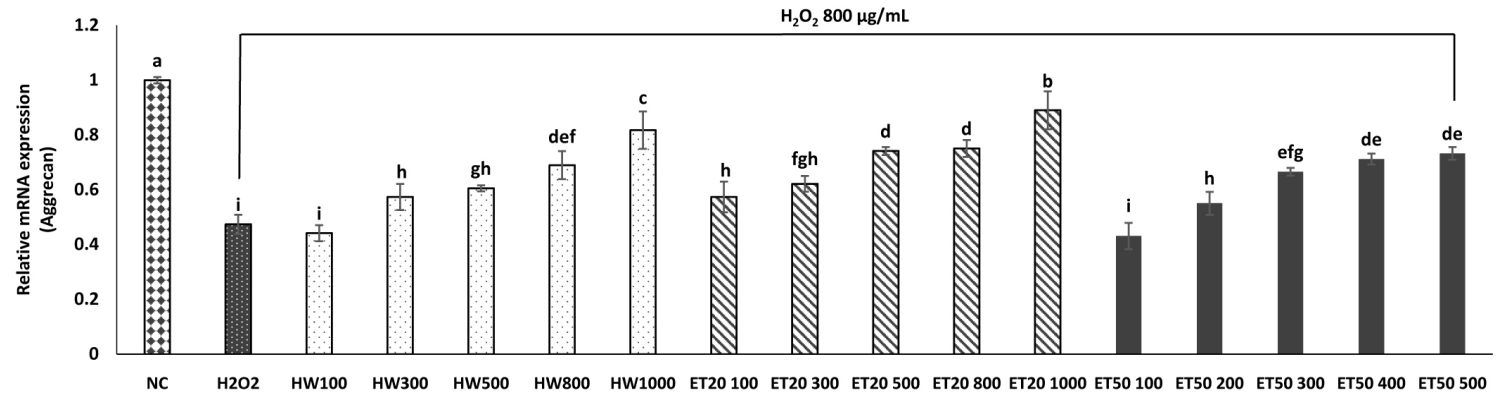

(D)

$\mathrm{H}_{2} \mathrm{O}_{2} 800 \mu \mathrm{g} / \mathrm{mL}$

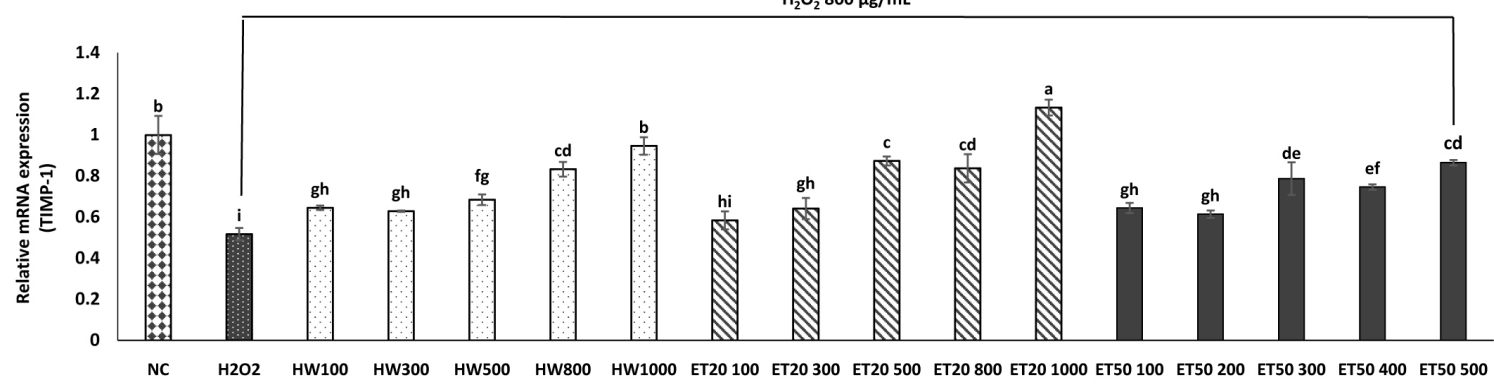

(E)

$\mathrm{H}_{2} \mathrm{O}_{2} 800 \mu \mathrm{g} / \mathrm{mL}$

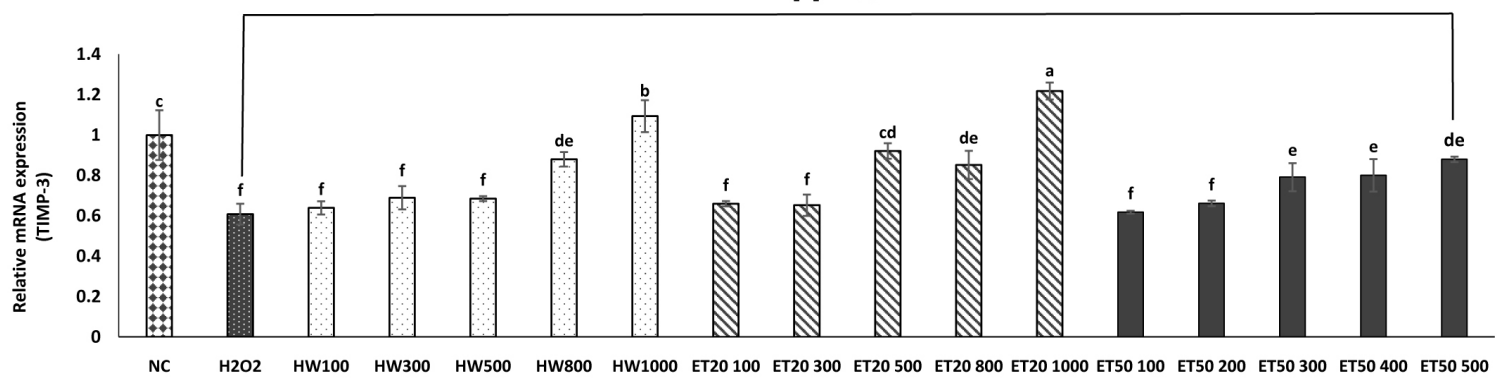

Fig. 4. Effect of HF extracts on genetic expression of anabolic factors in primary cultured cells. (A) Col-1, (B) Col-2, (C) Aggrecan, (D) TIMP-1, (E) TIMP-3, HW; Hot water extract 100-1,000 $\mu \mathrm{g} / \mathrm{mL}$, ET20; $20 \%$ EtOH extract 100-1,000 $\mathrm{\mu g} / \mathrm{mL}$, ET50; 50\% EtOH extract 100-1,000 $\mu \mathrm{g} / \mathrm{mL}$. Data are expressed as mean \pm SD $(n=8 / \mathrm{group})$. Significant differences were determined using Duncan's multiple range test at $P<0.05$. 
(A) $\mathrm{H}_{2} \mathrm{O}_{2} 800 \mu \mathrm{g} / \mathrm{mL}$

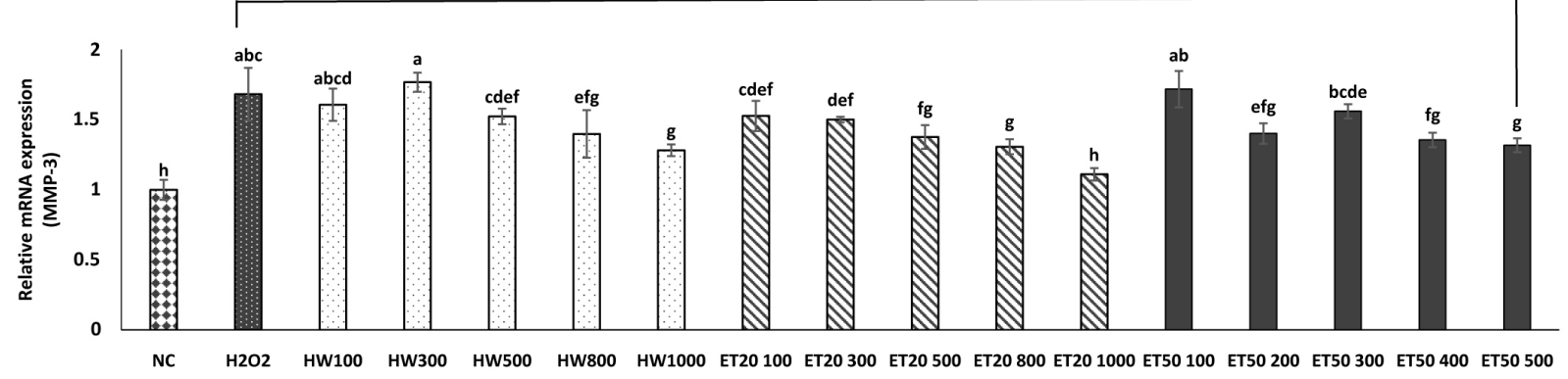

(B)

$\mathrm{H}_{2} \mathrm{O}_{2} 800 \mu \mathrm{g} / \mathrm{mL}$

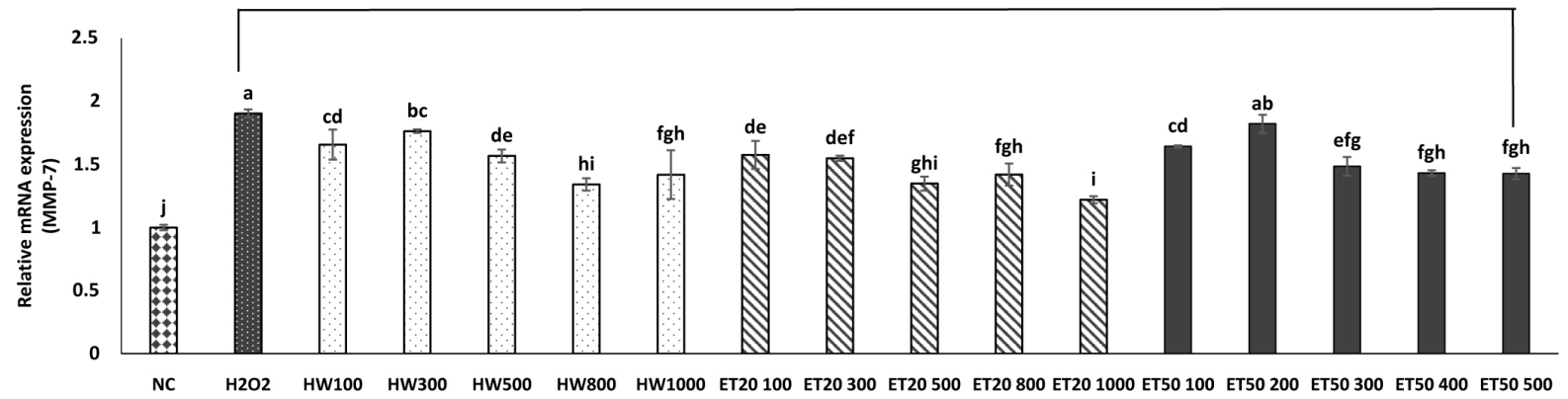

Fig. 5. Effect of HF extracts on genetic expression of catabolic factors in primary cultured cells. (A) MMP-3, (B) MMP-7. HW; Hot water extract $100-1,000 \mu g / m L$, ET20; $20 \%$ EtOH extract 100-1,000 $\mu \mathrm{g} / \mathrm{mL}$, ET50; 50\% EtOH extract 100-1,000 $\mu \mathrm{g} / \mathrm{mL}$. Data are expressed as mean $\pm \mathrm{SD}$ ( $\mathrm{n}=8 / \mathrm{group}$ ). Significant differences were determined using Duncan's multiple range test at $P<0.05$.

and TIMP-3 was significantly decreased in $800 \mu \mathrm{M} \mathrm{H} \mathrm{H}_{2} \mathrm{O}_{2}$ treated-chondrocytes compared with normal chondrocytes. However, $\mathrm{H}_{2} \mathrm{O}_{2}$ treated-chondrocytes with HW, ET20, and ET50 showed a significant dose-dependent decrease in expression of type I collagen, type II collagen, aggrecan, TIMP-1, and TIMP-3 compared with that of $\mathrm{H}_{2} \mathrm{O}_{2}$ treated-chondrocytes. In particular, expression of type I collagen, type II collagen, aggrecan, TIMP-1, and TIMP-3 in 1,000 $\mu \mathrm{g} / \mathrm{mL}$ ET20-treated chondrocytes showed the most significant increase compared with that of $\mathrm{H}_{2} \mathrm{O}_{2}$ treated-chondrocytes $(P<0.05)$ (Fig. 4).

Expression of MMP-3 and MMP-7 was significantly increased in $800 \mu \mathrm{M} \mathrm{H}_{2} \mathrm{O}_{2}$-treated chondrocytes compared with normal chondrocytes. In contrast, expression of MMP-3 and MMP-7 in $1,000 \mu \mathrm{g} / \mathrm{mL}$ ET20-treated chondrocytes showed the most significant decrease compared with that of $\mathrm{H}_{2} \mathrm{O}_{2}$ treatedchondrocytes $(P<0.05)$ (Fig. 5).

We confirmed that the effect of ET20 could improve inflammation and degradation of matrix more than $\mathrm{HW}$ and ET50 in osteoarthritis-like chondrocytes. Therefore, we examined the effect of ET20 in an osteoarthritis-induced animal model.

Effects of Hijikia fusiforme on nitric oxide production in an osteoarthritis-induced animal model

To examine whether a HFE can modulate the inflammatory process by regulating the secretion of NO, we measured the serum levels of NO. As illustrated in Fig. 6, a substantial increase in the NO level was detected in the serum of the sham group on day 24 post-MIA injection. In contrast, the NO level in $\mathrm{RH}$ treated cells was significantly decreased by $1.63 \pm 0.01$ compared with the sham group. The HFE250 and HFE500 groups showed significantly decreased levels of NO in serum compared with

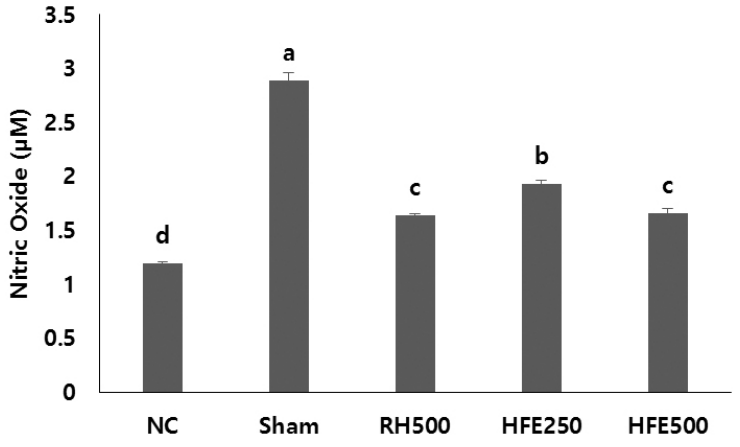

Fig. 6. Inhibitory effects of HFE on serum NO level. Normal control; Not injection + AIN 93G, Sham; Injection MIA + AIN 93G, RH500; Injection MIA + AIN 93G + Rose Hip extract $500 \mu \mathrm{g} / \mathrm{kg}$, HFE 250; Hijikia fusiforme 20\% EtOH extract $250 \mu \mathrm{g} / \mathrm{kg}$, HFE 500; Hijikia fusiforme $20 \%$ EtOH extract $500 \mu \mathrm{g} / \mathrm{kg}$. Data are expressed as mean $\pm \mathrm{SD}(\mathrm{n}=$ 8/group). Significant differences were determined using Duncan's multiple range test at $P<0.05$.

the sham group $(P<0.05)$ (Fig. 6).

Effects of Hijikia fusiforme on prostaglandin $E_{2}\left(P G E_{2}\right)$ production in an osteoarthritis-induced animal model

The $\mathrm{PGE}_{2}$ level in the serum of the sham group was significantly increased compared with that of the normal control group. Treatment with HFE500 resulted in a significant decrease in the $\mathrm{PGE}_{2}$ level in the serum compared with that in the sham group $(P<0.05)$ (Fig. 7).

Effects of Hijikia fusiforme on genetic expression of anabolic and catabolic factors in an osteoarthritis-induced animal model

Expression of type I collagen, type II collagen, and aggrecan 


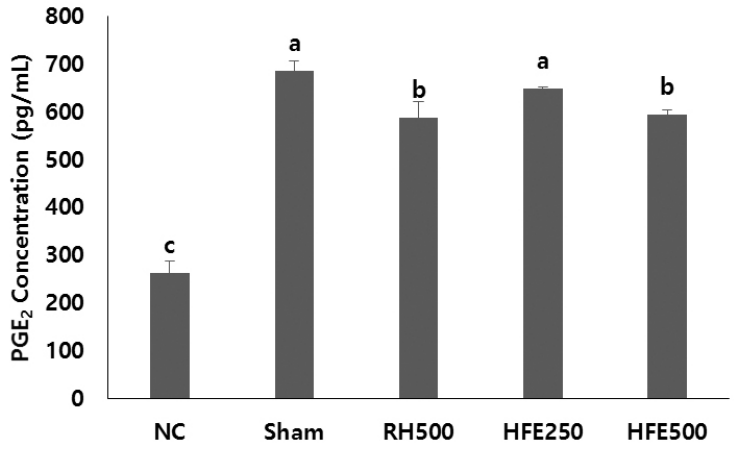

Fig. 7. Inhibitory effects of HFE on serum $\mathrm{PGE}_{2}$ level. Normal control; Not injection + AIN 93G, Sham; Injection MIA + AIN 93G, RH500; Injection MIA + AIN 93G + Rose Hip extract $500 \mu \mathrm{g} / \mathrm{kg}$, HFE 250; Hijikia fusiforme $20 \%$ EtOH extract $250 \mu \mathrm{g} / \mathrm{kg}$, HFE 500; Hijikia fusiforme $20 \%$ EtOH extract $500 \mu \mathrm{g} / \mathrm{kg}$. Data are expressed as mean $\pm \mathrm{SD}(\mathrm{n}=$ 8/group). Significant differences were determined using Duncan's multiple range test at $P<0.05$.

(A)

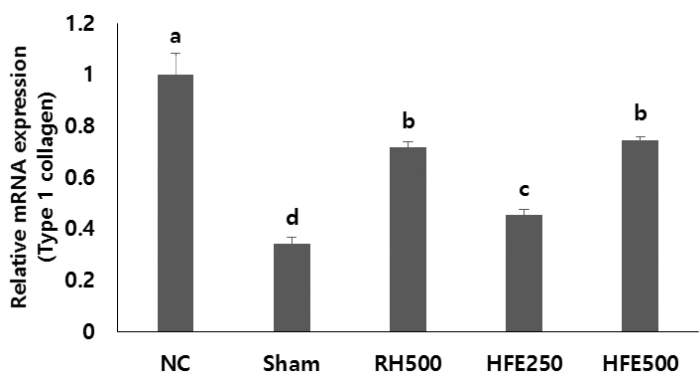

(B)

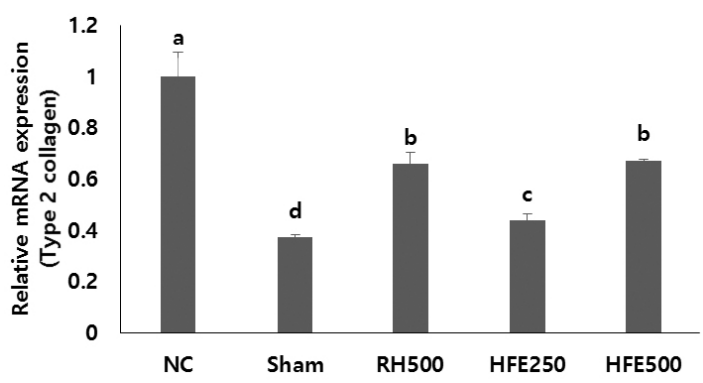

(C)

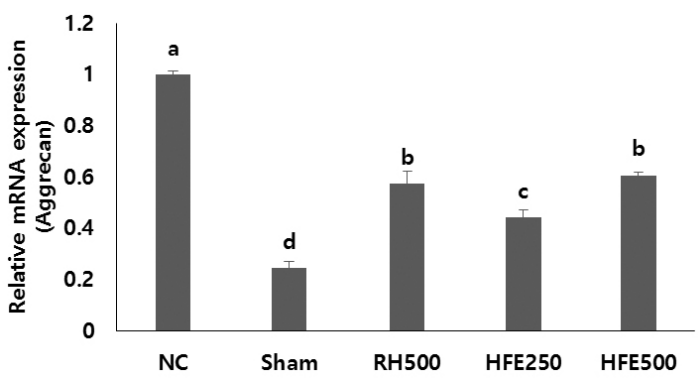

Fig. 8. Genetic expression of anabolic factors in MIA rat articular cartilage. Normal control; Not injection + AIN 93G, Sham; Injection MIA + AIN 93G, RH500; Injection MIA + AIN 93G + Rose Hip extract $500 \mu \mathrm{g} / \mathrm{kg}$, HFE 250; Hijikia fusiforme 20\% EtOH extract $250 \mu \mathrm{g} / \mathrm{kg}$, HFE 500; Hijikia fusiforme 20\% EtOH extract $500 \mu \mathrm{g} / \mathrm{kg}$. Data are expressed as mean $\pm S D$ ( $n=8 /$ group). Significant differences were determined using Duncan's multiple range test at $P<0.05$.

was significantly decreased in the sham group compared with that in the normal control group. In contrast, a significant increase in the expression of type I collagen, type II collagen, and aggrecan was observed in the HFE groups compared with
(A)

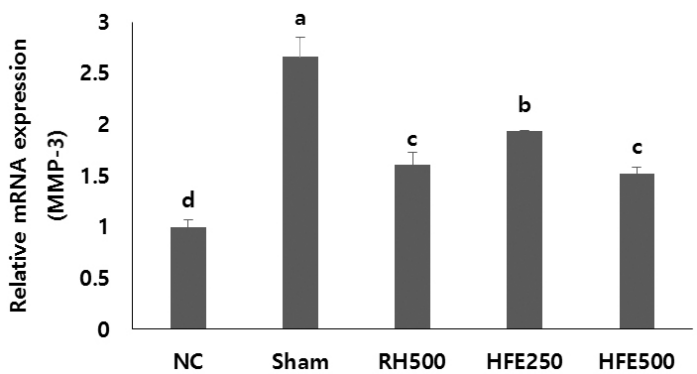

(B)

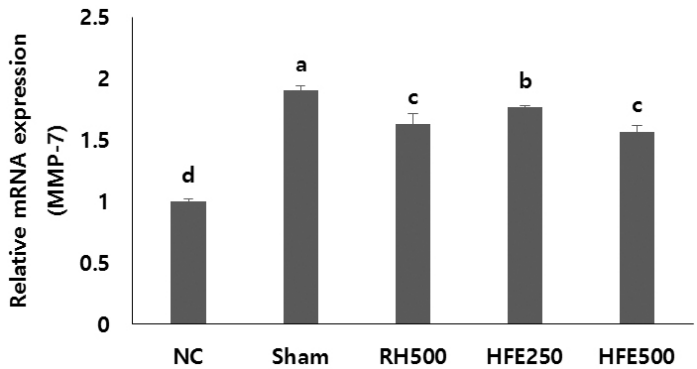

Fig. 9. Genetic expression of catabolic factors in MIA rat articular cartilage. Normal control; Not injection + AIN 93G, Sham; Injection MIA + AIN 93G, RH500; Injection $\mathrm{MIA}+\mathrm{AIN} 93 \mathrm{G}$ + Rose Hip extract $500 \mu \mathrm{g} / \mathrm{kg}$, HFE 250; Hijikia fusiforme $20 \% \mathrm{EtOH}$ extract $250 \mu \mathrm{g} / \mathrm{kg}$, HFE 500; Hijikia fusiforme $20 \%$ EtOH extract $500 \mu \mathrm{g} / \mathrm{kg}$. Data are expressed as mean $\pm S D$ ( $n=8 /$ group). Significant differences were determined using Duncan's multiple range test at $P<0.05$.

that of the sham group, and it increased in a dose-dependent manner $(P<0.05)$ (Fig. 8).

Expression of MMP-3 and MMP-7 was significantly increased in the sham group compared with that in the normal control group. Compared with the sham group, oral administration with HFE caused a significant dose-dependent decrease in expression of MMP-3 and MMP-7 $(P<0.05)$ (Fig. 9).

\section{DISCUSSION}

Progression of degenerative osteoarthritis is characterized by changes in extracellular matrix composition, including the degradation of collagen and aggrecan, which can induce the loss of articular cartilage. The major components of the extracellular matrix such as type I collagen, type II collagen, and aggrecan are synthesized and controlled by chondrocytes. Therefore, chondrocytes have been used in in vitro models for osteoarthritis experiments [17-19]. In the current study, LPS or $\mathrm{H}_{2} \mathrm{O}_{2}$ was used for induction of osteoarthritis-like inflammation in chondrocytes [17-19]. We confirmed that HW, ET20, and ET50 could improve inflammation and degradation of matrix in LPS or $\mathrm{H}_{2} \mathrm{O}_{2}$-induced chondrocytes through increases in the expression of type I collagen, type II collagen, aggrecan, TIMP-1, and TIMP-3 and decreases in expression of TNF-a, IL-6, MMP-3, and MMP-7.

Pro-inflammatory cytokines (IL-6 and TNF- $a$ ) in osteoarthritis affect the catabolism of cartilage tissue. Chondrocytes in osteoarthritis constitutively exhibit the activation of response for inflammation and the expression of MMPs to degradation of extracellular matrix proteins [20,21]. MMPs play an important role in the process in normal tissue of differentiation, wound healing, re-formation, organ formation, reproductive, blood 
vessel formation, tissue absorption and organization, and it is also expressed in pathological conditions such as arthritis for an important role in the pathogenesis [22]. Catrina et al. [20] showed that anti-TNF-a therapy significantly down-regulated serum levels of MMP-1 and MMP-3 and the ratio of MMPs to tissue inhibitor of metalloproteinase (TIMP)-1 in arthritis patients. Some studies have reported that IL- 6 induced bone resorption by stimulating the recruitment and formation of osteoclasts [21]. Houssiau et al. [21] reported that IL-6 activity was significantly elevated in synovial fluid from patients with arthritis or other inflammatory arthritides.

In this current study, we found a marked increase in the expression of IL- 6 and TNF- $\alpha$ in LPS-induced chondrocytes and in the expression of MMP-3 and MMP-7 in $\mathrm{H}_{2} \mathrm{O}_{2}$-induced chondrocytes. However, HW, ET20, and ET50 induced the repression of changes in the expression induced by LPS and $\mathrm{H}_{2} \mathrm{O}_{2}$. According to these reports and our results, we suggest that expression of matrix proteins such as collagens and aggrecan may improve by inhibiting the activation of response of pro-inflammatory cytokines.

Many studies have clearly indicated an important role of $\mathrm{PGE}_{2}$ and NO mediated by inflammation and pain in osteoarthritis [23-27]. NO, which is normally generated by iNOS, plays an important role in primary immune defense, and in physiological regulation such as blood vessel expansion [23]. However, when it is expressed excessively, NO is the factor that induces an inflammatory response and cellular necrosis. In addition, NO can cause dilation of blood vessels and increase the TNF- $a$ of permeability in the articulation. As a result, it induces the apoptosis mechanisms of cartilage cells [24]. PGE 2 , which is produced by such as TNF- $a$ in inflammatory reactions, is a factor involved in the development of articular cartilage degradation of osteoarthritis $[25,26]$. In addition, by forming the osteophytes of $\mathrm{PGE}_{2}$, it shows abnormal growth goals and pain, these symptoms are known to occur frequently in degenerative arthritis [27].

In the current study, we used the MIA injection-induced rat model for examination of effects of HFE extract on osteoarthritis. MIA injection disrupts chondrocyte metabolism and produces cartilage degeneration, which is similar to the histopathology of the degenerating joint seen in the human osteoarthritis [28]. We found that the $\mathrm{NO}$ and $\mathrm{PGE}_{2}$ levels were significantly increased in the serum of the sham group compared with that of the normal control group. In addition, in the sham group, the genetic expression of type I collagen, type II collagen, and aggrecan was significantly decreased and the expression of MMP-3 and MMP-7 was significantly increased in the sham group compared with that in the normal control group. However, oral administration of ET20 (HFE), the best effects in vitro assay, caused significant decreases in $\mathrm{NO}, \mathrm{PGE}_{2}$, MMP-3, and MMP-7 and significant increases in type I collagen, type II collagen, and aggrecan in a dose-dependent manner compared with that in the normal control group. The study by Kim et al. [29] reported that 5-hydroxy-3,6,7,8,3',4'-hexamethoxyflavone (5HHMF), a polyhydroxyflavone isolated from Hizikia fusiforme, inhibited the production of $\mathrm{PGE}_{2}, \mathrm{NO}$, and pro-inflammatory cytokines and decreased the transcriptional levels in LPS stimulated RAW 264.7 murine macrophage cells.
According to this study and our current results, we suggest that Hizikia fusiforme may improve inflammation, which may be attributed to the inhibition of production of $\mathrm{PGE}_{2}, \mathrm{NO}$, and pro-inflammatory cytokines.

In conclusion, Hijikia fusiforme $20 \%$ ethanol extract decreased the articular cartilage damage and development of osteoarthritis by preventing activation of inflammation in vitro and in vivo. This finding suggests that Hijikia fusiforme $20 \%$ ethanol extract might be considered as a potential therapeutic treatment for degenerative osteoarthritis.

\section{REFERENCES}

1. Ministry of Health and Welfare, Korea Centers for Disease Control and Prevention. Korea Health Statistics 2012: Korea National Health and Nutrition Examination Survey (KNHANES V-3). Cheongwon: Korea Centers for Disease Control and Prevention: 2013.

2. Statistics Korea. Senior Statistical Reports. Daejeon: Statistics Korea: 2010.

3. Garner BC, Stoker AM, Kuroki K, Evans R, Cook CR, Cook JL. Using animal models in osteoarthritis biomarker research. J Knee Surg 2011;24:251-64.

4. Nam DE, Kim OK, Shim TJ, Kim JH, Lee J. Effect of Boswellia serrata extracts on degenerative osteoarthritis in vitro and in vivo models. J Korean Soc Food Sci Nutr 2014;43:631-40.

5. Lee JH, Fitzgerald JB, Dimicco MA, Grodzinsky AJ. Mechanical injury of cartilage explants causes specific time-dependent changes in chondrocyte gene expression. Arthritis Rheum 2005;52:2386-95.

6. Roy S, Khanna S, Krishnaraju AV, Subbaraju GV, Yasmin T, Bagchi D, Sen CK. Regulation of vascular responses to inflammation: inducible matrix metalloproteinase-3 expression in human microvascular endothelial cells is sensitive to antiinflammatory Boswellia. Antioxid Redox Signal 2006;8:653-60.

7. Lee V, Cao L, Zhang Y, Kiani C, Adams ME, Yang BB. The roles of matrix molecules in mediating chondrocyte aggregation, attachment, and spreading. J Cell Biochem 2000;79:322-33.

8. Korea Health Industry Development Institute. Health Functional Food Industry Development Assistance Report 2011. Cheongwon: Korea Health Industry Development Institute 2011.

9. Jeon $\mathrm{MH}$, Kim M. Effect of Hijikia fusiforme Fractions on Proliferation and Differentiation in Osteoblastic MC3T3-E1 Cells. J Life Sci 2011;21:300-8.

10. Kim JA, Lee JM. The change of biologically functional compounds and antioxidant activities in Hizikia fusiformis with drying methods. Korean J Food Cult 2004;19:200-8.

11. Kapoor M, Martel-Pelletier J, Lajeunesse D, Pelletier JP, Fahmi H. Role of proinflammatory cytokines in the pathophysiology of osteoarthritis. Nat Rev Rheumatol 2011;7:33-42.

12. Ministry for Food, Agriculture, Forestry and Fisheries; Korea Food Research Institute. Development of Instant Health Foods Using Hijiki. Gwacheon: Ministry for Food, Agriculture, Forestry and Fisheries; 1997.

13. Watanabe T, Hirayama T, Takahashi T, Kokubo T, Ikeda M. Toxicological evaluation of arsenic in edible seaweed, Hizikia species. Toxicology 1979;14:1-22.

14. Dobashi $K$, Nishino T, Fujihara M, Nagumo T. Isolation and preliminary characterization of fucose-containing sulfated polysaccharides with blood-anticoagulant activity from the brown seaweed Hizikia 
fusiforme. Carbohydr Res 1989;194:315-20.

15. Holan ZR, Volesky B. Biosorption of lead and nickel by biomass of marine algae. Biotechnol Bioeng 1994;43:1001-9.

16. Jeong SC, Jeong YT, Lee SM, Kim JH. Immune-modulating activities of polysaccharides extracted from brown algae Hizikia fusiforme. Biosci Biotechnol Biochem 2015;79:1362-5.

17. Mathy-Hartert M, Martin G, Devel P, Deby-Dupont G, Pujol JP, Reginster JY, Henrotin Y. Reactive oxygen species downregulate the expression of pro-inflammatory genes by human chondrocytes. Inflamm Res 2003;52:111-8.

18. Asada S, Fukuda K, Oh M, Hamanishi C, Tanaka S. Effect of hydrogen peroxide on the metabolism of articular chondrocytes. Inflamm Res 1999;48:399-403.

19. Khan IM, Gilbert SJ, Caterson B, Sandell $\sqcup$, Archer CW. Oxidative stress induces expression of osteoarthritis markers procollagen IIA and $3 \mathrm{~B} 3(-)$ in adult bovine articular cartilage. Osteoarthritis Cartilage 2008;16:698-707.

20. Catrina Al, Lampa J, Ernestam S, af Klint E, Bratt J, Klareskog L, Ulfgren AK. Anti-tumour necrosis factor (TNF)-alpha therapy (etanercept) down-regulates serum matrix metalloproteinase (MMP)-3 and MMP-1 in rheumatoid arthritis. Rheumatology (Oxford) 2002;41:484-9.

21. Houssiau FA, Devogelaer JP, Van Damme J, de Deuxchaisnes CN, Van Snick J. Interleukin-6 in synovial fluid and serum of patients with rheumatoid arthritis and other inflammatory arthritides. Arthritis Rheum 1988;31:784-8.

22. Tchetverikov I, Lohmander LS, Verzijl N, Huizinga TW, TeKoppele JM, Hanemaaijer R, DeGroot J. MMP protein and activity levels in synovial fluid from patients with joint injury, inflammatory arthritis, and osteoarthritis. Ann Rheum Dis 2005;64:694-8.

23. Wink DA, Hines $H B$, Cheng RY, Switzer $C H$, Flores-Santana W, Vitek MP, Ridnour LA, Colton CA. Nitric oxide and redox mechanisms in the immune response. J Leukoc Biol 2011;89:873-91.

24. Thomas CM, Fuller CJ, Whittles CE, Sharif M. Chondrocyte death by apoptosis is associated with the initiation and severity of articular cartilage degradation. Int J Rheum Dis 2011;14:191-8.

25. Vane JR, Bakhle YS, Botting RM. Cyclooxygenases 1 and 2. Annu Rev Pharmacol Toxicol 1998;38:97-120.

26. Bensen WG, Fiechtner JJ, McMillen Jl, Zhao WW, Yu SS, Woods EM, Hubbard RC, Isakson PC, Verburg KM, Geis GS. Treatment of osteoarthritis with celecoxib, a cyclooxygenase-2 inhibitor: a randomized controlled trial. Mayo Clin Proc 1999;74:1095-105.

27. Hardy MM, Seibert K, Manning PT, Currie MG, Woerner BM, Edwards $D$, Koki A, Tripp CS. Cyclooxygenase 2-dependent prostaglandin $E_{2}$ modulates cartilage proteoglycan degradation in human osteoarthritis explants. Arthritis Rheum 2002;46:1789-803.

28. Combe R, Bramwell S, Field MJ. The monosodium iodoacetate model of osteoarthritis: a model of chronic nociceptive pain in rats? Neurosci Lett 2004;370:236-40.

29. Kim MJ, Lee HH, Jeong JW, Seo MJ, Kang BW, Park JU, Kim KS, Cho YS, Seo Kl, Kim GY, Kim Jl, Choi YH, Jeong YK. Anti-inflammatory effects of 5-hydroxy-3,6,7,8,3',4'-hexamethoxyflavone via NF- $k B$ inactivation in lipopolysaccharide-stimulated RAW 264.7 macrophage. Mol Med Rep 2014;9:1197-203. 